\title{
Mycobacterium riyadhense overlooked: we can only find what we are looking for
}

\author{
Sahal Al-Hajoj ${ }^{1}$, Bright Varghese ${ }^{1}$, Jakko van Ingen ${ }^{2}$ and Dick van Soolingen ${ }^{2,3}$ \\ ${ }^{1}$ Mycobacteriology Research Section, Department of Infection and Immunity, King Faisal Specialist Hospital and \\ Research Centre, Riyadh, Saudi Arabia \\ ${ }^{2}$ Department of Medical Microbiology, Radboud University Nijmegen Medical Center, Nijmegen, The Netherlands \\ ${ }^{3}$ National Mycobacteria Reference Laboratory, Laboratories for Infectious Diseases and Prenatal Screening, \\ National Institute for Public Health and the Environment, Bilthoven, The Netherlands
}

Key words: Mycobacterium riyadhense; tuberculosis; mycobacteria

J Infect Dev Ctries 2013; 7(3):293-294.

(Received 05 February 2013 - Accepted 01 March 2013)

Copyright (C) 2013 Al-Hajoj et al. This is an open-access article distributed under the Creative Commons Attribution License, which permits unrestricted use, distribution, and reproduction in any medium, provided the original work is properly cited.

In 2009, we identified a novel mycobacterial species, Mycobacterium riyadhense, which was isolated from a patient in Riyadh, Saudi Arabia [1]. In 2012, there were two new reports on three additional cases in Korea, Bahrain and France, caused by the same bacteria, thus showing its emerging potential worldwide [2-3]. In these three cases, $M$. riyadhense was isolated from broncho-alveolar lavage and sputum, and associated with disease. All of the reported patients received regular anti-tuberculosis treatment until the causative agent was properly identified; for some, these results became available one year after presentation [3].

$M$. riyadhense is a slow-growing Mycobacterium which produces rough, white colonies after four weeks of incubation at $36^{\circ} \mathrm{C}$. Biochemical properties include (i) nitrate reduction, Tween 80 hydrolysis, arylsulfatase, and urease activities; (ii) absence of niacin accumulation, heat stable catalase and tellurite reduction; (iii) no growth on MacConkey agar; (iv) negative tolerance to $p$-nitrobenzoic acid, hydroxylamine and oleic acid; and (v) tolerance to thiophene-2-carboxylic hydrase, thiocetazone and isoniazid.

Phylogenetically, $M$. riyadhense is very close to $M$. szulgai and contains the region of difference-1 (RD1) with virulent factors (esat-6 and $c f p-10$ genes), also present in $M$. tuberculosis. The $16 \mathrm{~S}$ rRNA, hsp65 and $r p o B$ genes and the $16 \mathrm{~S}-23 \mathrm{~S}$ internal transcribed spacer sequences are unique [1]. The emerging reports on diseases mimicking tuberculosis (TB), but caused by $M$. riyadhense, emphasize the need for proper identification of mycobacteria in the diagnosis of mycobacterial diseases. It is conceivable that nontuberculous mycobacterial (NTM) diseases are more frequent than previously assumed and misdiagnosed as TB. This is especially a problem for bacteria such as $M$. riyadhense with its ability to cause pulmonary and extra pulmonary, TB-like disease in apparently immunocompetent individuals and which are actually identified as $M$. tuberculosis by some popular commercial identification assays such as GenoType MTBC/CM [1,3-4].

Proper distinction of TB and NTM disease is of the utmost importance, because this has profound consequences for treatment. Standardized TB treatment is insufficient for NTM, including $M$. riyadhense; currently reported cases followed at least nine months of first-line drug therapy to attain a cure [1-3]. The problem with the identification of newly recognized mycobacteria such as $M$. riyadhense is that there are no commercially available kits to facilitate a timely and accurate diagnosis; only DNA sequencing will offer definitive identification. The currently available identification assays focus on the most frequently isolated species and lack the flexibility to adjust to new and emerging species. If $M$. riyadhense infections are becoming more associated with serious disease, it would be important to include testing for these bacteria in commercial identification kits. Only frequent testing for $M$. riyadhense will provide an accurate assessment of its true prevalence. This 
species may be of specific interest because it harbours an ESX-1 system, a well-known virulence factor of $M$. tuberculosis that is also functional in NTM, at least in M. kansasii and M. szulgai [5].

More cases of TB than currently assumed may actually represent NTM disease, caused by species such as $M$. riyadhense; therefore, more sophisticated identification tools, including DNA sequencing and the inclusion of newly emerging species into commercial identification kits, are imperative research goals.

\section{Acknowledgements}

We dedicate this work to Professor Giovanni Fadda in his honor.

\section{References}

1. van Ingen J, Al-Hajoj SA, Boeree M, Al-Rabiah F, Enaimi M, de Zwaan R, Tortoli E, Dekhuijzen R, van Soolingen D (2009) Mycobacterium riyadhense sp. nov., a non-tuberculous species identified as Mycobacterium tuberculosis complex by a commercial line-probe assay. Int J Syst Evol Microbiol 59: 1049-1053.

2. Choi JI, Lim JH, Kim SR, Lee SH, Park JS, Seo KW, Jeon JB, Jeong J (2012) Lung infection caused by Mycobacterium riyadhense confused with Mycobacterium tuberculosis: the first case in Korea. Ann Lab Med 32: 298-303.
3. Godreuil S, Marchandin H, Michon AL, Ponsada M, Chyderiotis G, Brisou P, Bhat A, Panteix G (2012) Mycobacterium riyadhense pulmonary infection, France and Bahrain. Emerg Infect Dis 18: 176-178.

4. Tortoli E, Pecorari M, Fabio G, Messinò M, Fabio A (2010) Commercial DNA probes for Mycobacteria incorrectly identify a number of less frequently encountered species. J Clin Microbiol 48: 307-310.

5. Houben D, Demangel C, van Ingen J, Perez J, Baldeón L, Abdallah AM, Caleechurn L, Bottai D, van Zon M, de Punder K, van der Laan T, Kant A, Bossers-de Vries R, Willemsen P, Bitter W, van Soolingen D, Brosch R, van der Wel N, Peters PJ (2012) ESX-1-mediated translocation to the cytosol controls virulence of mycobacteria. Cell Microbiol 14: 12871298.

\section{Corresponding author}

Sahal Al-Hajoj

Mycobacteriology Research Section

Department of Infection and Immunity

King Faisal Specialist Hospital and Research Centre PB NO-3354

Riyadh, Saudi Arabia, 11211

Telephone -00966-1-4424992, Fax: 00966-1-4627858

Email: hajoj@kfshrc.edu.sa

Conflict of interests: No conflict of interests is declared. 